\title{
An Approximate Model Based on Feature Matrix for Nonlinear Hybrid Systems
}

\author{
Shihan Yang ${ }^{1,2}$ and Qiong $\mathrm{Yu}^{3}$ \\ ${ }^{1}$ Faculty of Management and Economics, Kunming University of Science and Technology, Kunming, China \\ ${ }^{2}$ College of Software and Information Security, Guangxi University for Nationalities, Nanning, China \\ ${ }^{3}$ College of Sciences, Guangxi University for Nationalities, Nanning, China
}

\begin{abstract}
Nonlinear hybrid systems are focused on. A modeling framework based on the feature matrix, which is a matrix of Boolean function of the truth table, is proposed. Then, we study a special kind of nonlinear hybrid systems. This kind of systems is linearized by the means of Taylor series expansion, where linearizing parameters can be avoided due to all elements of the feature matrix are zero or one, which leads not to linearize each location of the model.
\end{abstract}

Keywords-nonlinear hybrid systems; feature matrix; truth table; linearizing

\section{INTRODUCTION}

Hybrid systems may involve both continuous and discrete states with continuous and discrete inputs. The term hybrid has also been used to describe processes that involve continuous dynamics and discrete (logical) decision [1, 2, 3]. Typically, the flow-field describing the evolution of continuous states is dependent on discrete phenomena characterized by discrete state event as well as control events due to discrete inputs. A formal description of hybrid systems may be represented by a hybrid automaton $[4,5]$. The choice of the specific model structure of the hybrid automaton depends on the application at hand. Control applications, fault detection and diagnosis studies have led to development of various linear modeling paradigms such as piecewise affine [6] (PWA) and mixed logical dynamic [2] (MLD) models. The equivalence between MLD and a variety of other linear hybrid models has also been proven [7].

In the area of nonlinear hybrid systems, Buss et al. [8] presented the Hybrid State Model (HSM), which models the nonlinear dynamics of the system with discrete states as well as discrete control inputs. Furthermore, Naresh et al. [9] developed the multiple partially linearized modeling framework corresponding to all locations, which can represent arbitrary nonlinear hybrid dynamic systems while retaining a fixed model structure. Thomas et al. [10] extended the modelchecking approach to the analysis of nonlinear hybrid systems, by reduction to the linear problem. The approximation of hybrid systems using linear hybrid automata has been advocated previously. Two translation techniques from nonlinear to linear hybrid automata are suggested in [10]. Thomas et al. [11] improved on the rate translation technique by suggesting linear phase-portrait approximations that are strictly more accurate.
The modified hybrid systems have the parameters which lead to the complex model of linearization. In particular, we present a framework of nonlinear hybrid systems that avoids parameters of each location. There are many kinds of nonlinear hybrid systems, so linearizing the model of nonlinear hybrid systems requires classification. Then, we study a special kind of nonlinear hybrid systems which can be seen as the feature matrix's linear function. Furthermore, we linearize the model by mean of Taylor series, which just right produces the feature matrix that can avoid parameters.

This paper is organized as follows. In section 2, the modified hybrid state model is reviewed. In section 3 and 4 , modeling framework based on feature matrix is proposed. In section 5, we study a special nonlinear hybrid system and linearize it. Section 6 gives conclusion.

\section{MODELING FRAMEWORK FOR NONLINEAR HYBRID SYSTEMS}

The recent interest in nonlinear hybrid dynamical systems have forced the merger of two very different modeling and control methodologies, namely those for discrete and for continuous systems. In this section, the modified hybrid state model is reviewed for the modeling of fairly general class of nonlinear hybrid dynamical systems. The model is related to literatures [8, 12, 13]. The modified hybrid state model [9] can be used for modeling and control of nonlinear hybrid systems. The modified hybrid state model can be written as follows:

$$
\begin{gathered}
\dot{\mathbf{x}}^{c}=f_{g}\left(\mathbf{x}^{c}, \mathbf{u}^{c}, \delta\right) \\
\mathbf{x}^{d}(t)=\mathbf{b}_{d}(\delta)(t) \\
\mathbf{E}_{1} \mathbf{u}^{c}(t)+\mathbf{E}_{2} \delta(t)+\mathbf{E}_{3} \mathbf{x}(t) \leq \mathbf{E}_{4} \\
\mathbf{x}^{c}\left(t^{+}\right)=\mathbf{x}^{c}\left(t^{-}\right) \\
\mathbf{x}^{d}\left(t^{+}\right)=\mathbf{b}_{d}\left(\delta\left(t^{+}\right)\right)
\end{gathered}
$$


where $\mathbf{x}^{c}(t), \mathbf{x}^{d}(t)$ are the continuous state and discrete state, respectively; $\mathbf{u}^{d}(t)$ is the discrete inputs; $\delta$ with binary indicator variables $\delta_{i} \in\{0, l\}$ is the indicator vector; the elements of $\mathbf{b}_{d}$ depend on the status of the vector $\delta(t)$; (1) and (2) describe the continuous state and discrete state respectively; (3) describes the constraint conditions, where $E_{i}(i=1,2,3,4)$ are the corresponding coefficient matrices of appropriate size; (4) describes that the continuous states are reset after an event. Symbol $t$ indicates that a function equals zero and it will result in a sign change, that is a positive value at $t^{-}$will become negative at $t^{+}$after equaling zero and vice-versa.

Typically, models derived from real systems will be wellposed and solution of (1) - (5) will result in a unique trajectory $\mathbf{x}(t)$, for a given initial condition and control input trajectory [2]. However, use of the above modified hybrid state model for a models based receding horizon control scheme [14] needs solution of a MINLP optimization problem online. There are two main difficulties about MINLP optimization problem as the following: firstly, optimization process is easy to fall into local optimal solution which leads to increase the difficulty of the optimization; second, the modified hybrid system contains binary variables $\delta$ and continuous variables $\mathbf{x}^{c}(t), \mathbf{u}^{c}(t)$. Thus hinders the optimal solution. To overcome these difficulties, we require that binary and continuous variables cannot appear simultaneously in the modified hybrid system. But continuous variables of the modified hybrid system are indispensable, so the binary variables of the modified hybrid system are changed. Further, the future prediction will require numerous integrations of (1).

\section{THE FEATURE MATRIX OF BOOLEAN FUNCTION}

Boolean function [15] is the study of the logical operation between variable 0 and variable 1 . One of the basic stages in discrete logic design is the representation of Boolean functions by various formulas in a given basis $[16,17,18,19]$. It has many representation methods, such as list method, algebraic normal form representation, Walsh spectral representation [20], matrix representation and single variable representation. Matrix representation is one of the important representation methods of Boolean function. Essentially, Matrix representation is the feature matrix of Boolean function.

Definition 3.1 [21] Let $f\left(x_{1}, x_{2}, \cdots, x_{n}\right) \in \mathbf{B}_{n}, \alpha \in \mathbf{F}_{2}^{n}$, if $f(\alpha)=1$, then $\alpha$ is a feature vector of Boolean function $f(\mathbf{x})$, the set of all feature vectors is called support set. The set of all feature vectors is noted as $\mathbf{1}_{f}$ :

$$
\mathbf{1}_{f}=\left\{\alpha \mid f(\alpha)=1, \alpha \in \mathbf{F}_{2}^{n}\right\}
$$

where $f\left(x_{1}, x_{2}, \cdots, x_{n}\right)$ is the set of Boolean functions, $\mathbf{F}_{2}=\{0,1\}, \mathbf{F}_{2}$ represents $n$-dimensional vector space of $\mathbf{F}_{2}$.

$\left|\mathbf{1}_{f}\right|=w$ represents the number of elements in the set $\mathbf{1}_{f}$ which can be called the weight of Boolean function $f(x)$. The $w$ vectors in the support set $\mathbf{1}_{f}$ can be with the dictionary order from large to small, let the $i-t h$ vector be $w_{i}=\left(c_{i 1}, c_{i 2}, \cdots, c_{i n}\right), 1 \leq i \leq w$. Let

$$
\mathbf{C}_{f}=\left(\begin{array}{cccc}
c_{11} & c_{12} & \cdots & c_{1 n} \\
c_{21} & c_{22} & \cdots & c_{2 n} \\
\vdots & \vdots & \vdots & \vdots \\
c_{w 1} & c_{w 2} & \cdots & c_{w n}
\end{array}\right),
$$

the matrix $\mathbf{C}_{f}$ can be called the feature matrix of Boolean function $f(\mathbf{x})$.

It should be noted, without considering the row permutation of the feature matrix, there is one relationship between Boolean function and its feature matrix. Thus, we don't consider the row permutation of the feature matrix in this paper.

\section{Modeling Framework Based On Feature Matrix}

Theoretical work on controllability properties of nonlinear hybrid systems has attracted the interest of many scholars. Literature [22] has been resolved several problems of low state and control dimension of nonlinear hybrid systems.

The modified hybrid state model (1) consists of three variables, i.e. a continuous state $\mathbf{x}^{c}$, a continuous manipulated inputs $\mathbf{u}^{c}$ and an indicator variable $\delta$.

Because an indicator variable $\delta$ is used to identify occurrence of an event, the new location, the indicator variable $\delta$ decides the evolution map of continuous states. And $\delta=\left(\delta_{1}, \delta_{2}, \cdots, \delta_{n_{s}}\right)^{T}, \quad \delta_{i} \in\{0,1\}\left(i=1,2, \cdots, n_{s}\right)$ is binary indicator variables, all elements of the indicator variable $\delta=\left(\delta_{1}, \delta_{2}, \cdots, \delta_{n_{s}}\right)^{T}$ are 0 or 1 . So the indicator variable $\delta=\left(\delta_{1}, \delta_{2}, \cdots, \delta_{n_{s}}\right)^{T}$ may be seen as a truth table. According to the truth table, appropriate Boolean function can be constructed. We assume that the appropriate Boolean function of an indicator variable $\delta=\left(\delta_{1}, \delta_{2}, \cdots, \delta_{n_{s}}\right)^{T}$ is $p\left(\delta_{1}, \delta_{2}, \cdots, \delta_{n_{s}}\right)$, where $\left(\delta_{1}, \delta_{2}, \cdots, \delta_{n_{s}}\right)$ is the binary representation of $\delta$. Thus, one may define appropriate Boolean function $p\left(\delta_{1}, \delta_{2}, \cdots, \delta_{n_{s}}\right)$ corresponding to the indicator vector $\delta=\left(\delta_{1}, \delta_{2}, \cdots, \delta_{n_{s}}\right)^{T}$ as follows:

$$
\delta=\left(\delta_{1}, \delta_{2}, \cdots, \delta_{n_{s}}\right)^{T} \leftrightarrow p\left(\delta_{1}, \delta_{2}, \cdots, \delta_{n_{s}}\right)
$$

Thus, the continuous state evolution map of the modified hybrid state model (1) may be rewritten as,

$$
\dot{\mathbf{x}}^{c}=f_{g}\left(\mathbf{x}^{c}, \mathbf{u}^{c}, p\left(\delta_{1}, \delta_{2}, \cdots, \delta_{n_{s}} £\right)\right.
$$

where $f_{g}$ is a global flow-field that subsumes all locationdependent flow-fields $f_{l}$ and is controlled by the Boolean function $p\left(\delta_{1}, \delta_{2}, \cdots\right), \delta_{n_{s}}$, which is determined by (6).

According to Boolean function, there may be a corresponding feature matrix of Boolean function. We assume that the corresponding feature matrix of the Boolean function $p\left(\delta_{1}, \delta_{2}, \cdots, \delta_{n_{s}}\right)$ is $\mathbf{C}_{p}$. Thus, information characterized by Boolean function $p\left(\delta_{1}, \delta_{2}, \cdots, \delta_{n_{s}}\right)$ is the same as that of its feature matrix. This means Boolean function $p\left(\delta_{1}, \delta_{2}, \cdots, \delta_{n_{s}}\right)$ and its feature matrix $\mathbf{C}_{p}$ are equivalent. So, one may construct 
appropriate feature $\mathbf{C}_{p}$ corresponding to the Boolean function $p\left(\delta_{1}, \delta_{2}, \cdots, \delta_{n_{s}}\right)$ as follows:

$$
p\left(\delta_{1}, \delta_{2}, \cdots, \delta_{n_{s}}\right) \leftrightarrow C_{p}
$$

Thus, the continuous state evolution map of the modified hybrid state model (1) may be rewritten as,

$$
\dot{\mathbf{x}}^{c}=\dot{\mathbf{x}}^{c}=f_{g}\left(\mathbf{x}^{c}, \mathbf{u}^{c}, \mathbf{C}_{p}\right)
$$

Because truth tables $\delta=\left(\delta_{1}, \delta_{2}, \cdots, \delta_{n_{s}}\right)^{T}$ and its Boolean function $p\left(\delta_{1}, \delta_{2}, \cdots, \delta_{n_{s}}\right)$ characterize the same information of occurrence of an event, Boolean function and its feature matrix $\mathbf{C}_{p}$ are equivalent; truth table $\delta=\left(\delta_{1}, \delta_{2}, \cdots, \delta_{n_{s}}\right)^{T}$ and the feature matrix $\mathbf{C}_{p}$ are equivalent. This means that information characterized by truth table $\delta=\left(\delta_{1}, \delta_{2}, \cdots, \delta_{n_{s}}\right)^{T}$ is equivalent to the information responded by feature matrix $\mathbf{C}_{p}$. That is, one may define an appropriate feature matrix $\mathbf{C}_{p}$ corresponding to the indicator vector $\delta=\left(\delta_{1}, \delta_{2}, \cdots, \delta_{n_{s}}\right)^{T}$ as follows:

$$
\delta \leftrightarrow \mathbf{C}_{p}
$$

A change in the status of feature matrix $\mathbf{C}_{p}$ corresponds to an event that may be triggered by discontinuity in states or discontinuity in inputs. Thus, the corresponding discrete states of hybrid systems may be rewritten as

$$
\mathbf{x}^{d}(t)=\mathbf{b}_{d}\left(\mathbf{C}_{p}\right)
$$

where the elements of $\mathbf{b}_{d}$ depend on the feature matrix $\mathbf{C}_{p}$. Due to the relation between truth table $\delta=\left(\delta_{1}, \delta_{2}, \cdots, \delta_{n_{s}}\right)$ and feature matrix $\mathbf{C}_{p}$, the equivalent representation in (3) may be reformulated as follows:

$$
\mathbf{E}_{1} \mathbf{u}^{c}(t)+\mathbf{E}_{2} \mathbf{C}_{p}+\mathbf{E}_{3} \mathbf{x}(t) \leq \mathbf{E}_{4}
$$

where $\mathbf{E}_{i}(i=1,2,3,4)$ are the corresponding coefficient matrix of appropriate size.

Finally, the modified hybrid state model (1) - (5) can be written as follows:

$$
\begin{aligned}
& \dot{\mathbf{x}}^{c}=f_{g}\left(\mathbf{x}^{c}, \mathbf{u}^{c}, \mathbf{C}_{p}\right) \\
& \mathbf{x}^{d}(t)=\mathbf{b}_{d}\left(\mathbf{C}_{p}\right) \\
& \mathbf{E}_{1} \mathbf{u}^{c}(t)+\mathbf{E}_{2} \mathbf{C}_{p}+\mathbf{E}_{3} \mathbf{x}(t) \leq \mathbf{E}_{4} \\
& \mathbf{x}^{c}\left(t^{+}\right)=\mathbf{x}^{c}\left(t^{-}\right)
\end{aligned}
$$

$$
\mathbf{x}^{d}=\mathbf{b}_{d}\left(\mathbf{C}_{p}\right)
$$

The HSM represented in (13) - (17) modifies the modified hybrid state model in (1) - (5) according to the truth table and its feature matrix, which may be used for modeling and control of nonlinear hybrid systems.

\section{MODEL OF LINEARIZATION}

Taylor series expansion linearization method is a kind of approximate linearization method because of the error caused by giving up some items. The basic idea of Taylor series expansion linearization is linear approximation nonlinear system in the vicinity of the equilibrium point. The HSM represented in (13) determines the type of nonlinear hybrid systems. That is, different (13) determines the different nonlinear hybrid systems. Nonlinear hybrid systems are more complex than linear hybrid systems. Thus, we can study nonlinear hybrid systems by linearizing nonlinear hybrid systems. Now, we consider a special nonlinear hybrid system. That is, (13) has the following type:

$$
\dot{\mathbf{x}}^{c}=h\left(\mathbf{x}^{c}, \mathbf{u}^{c}\right)+\mathbf{C}_{p} m\left(\mathbf{x}^{c}, \mathbf{u}^{c}\right)
$$

where

$$
h\left(\mathbf{x}^{c}, \mathbf{u}^{c}\right)=\left(\begin{array}{c}
h_{1}\left(x_{1}^{c}, u_{1}^{c}\right) \\
h_{2}\left(x_{2}^{c}, u_{2}^{c}\right) \\
\vdots \\
h_{n_{s}}\left(x_{n_{s}}^{c}, u_{n_{s}}^{c}\right)
\end{array}\right), m\left(\mathbf{x}^{c}, \mathbf{u}^{c}\right)=\left(\begin{array}{c}
m_{1}\left(x_{1}^{c}, u_{1}^{c}\right) \\
m_{2}\left(x_{2}^{c}, u_{2}^{c}\right) \\
\vdots \\
m_{n_{s}}\left(x_{n_{s}}^{c}, u_{n_{s}}^{c}\right)
\end{array}\right) .
$$

We assume that $\left(\mathbf{x}^{c}, \mathbf{u}^{c}\right)$ is the equilibrium point of $\dot{\mathbf{x}}^{c}=h\left(\mathbf{x}^{c}, \mathbf{u}^{c}\right)+\mathbf{C}_{p} m\left(\mathbf{x}^{c}, \mathbf{u}^{c}\right)$.

Performing a Taylor series expansion on $h\left(\mathbf{x}^{c}, \mathbf{u}^{c}\right)$ and $m\left(\mathbf{x}^{c}, \mathbf{u}^{c}\right)$ around the point $\left(\mathbf{x}^{c}, \mathbf{u}^{c}\right)$, one may obtain a linearized equation as follows, respectively:

$$
h\left(\mathbf{x}^{c}, \mathbf{u}^{c}\right)=\mathbf{A} \mathbf{x}^{c}(t)+\mathbf{B} \mathbf{u}^{c}(t),
$$

where $\mathbf{A} \in \mathfrak{R}^{n_{s} \times n_{s}}, \mathbf{B} \in \mathfrak{R}^{n_{s} \times n_{s}}$, and

$$
\begin{gathered}
\mathbf{x}^{c}(t)=\left(\begin{array}{c}
x_{1}^{c} \\
x_{2}^{c} \\
\vdots \\
x_{n_{s}}^{c}
\end{array}\right), \mathbf{u}^{c}(t)=\left(\begin{array}{c}
u_{1}^{c} \\
u_{2}^{c} \\
\vdots \\
u_{n_{s}}^{c}
\end{array}\right) . \\
\left(\mathbf{x}^{c}, \mathbf{u}^{c}\right)=\mathbf{C x}^{c}(t)+\mathbf{D u}^{c}(t),
\end{gathered}
$$

where $\mathbf{C} \in \mathfrak{R}^{n_{s} \times n_{s}}, \mathbf{D} \in \mathfrak{R}^{n_{s} \times n_{s}}$.

Thus we can get, 


$$
\begin{aligned}
\mathbf{C}_{P} m\left(\mathbf{x}^{c}, \mathbf{u}^{c}\right) & =\mathbf{C}_{p}\left(\mathbf{C x}^{c}(t)+\mathbf{D u}^{c}(t)\right) \\
& =\mathbf{C}_{p} \mathbf{C} \mathbf{x}^{c}(t)+\mathbf{C}_{p} \mathbf{D u}^{c}(t)
\end{aligned}
$$

Let $\mathbf{A}_{1}=\mathbf{C}_{p} \mathbf{C}, \mathbf{B}_{1}=\mathbf{C}_{p} \mathbf{D}$. All elements of the feature matrix $\mathbf{C}_{p}$ are 0 or 1 . So,

$$
\begin{aligned}
\mathbf{C}_{p} m\left(\mathbf{x}^{c}, \mathbf{u}^{c}\right) & =\mathbf{C}_{p} \mathbf{C} \mathbf{x}^{c}(t)+\mathbf{C}_{p} \mathbf{D} \mathbf{u}^{c}(t) \\
& =\mathbf{A}_{l} \mathbf{x}^{c}(t)+\mathbf{B}_{l} \mathbf{u}^{c}(t)
\end{aligned}
$$

Finally, we can obtain a linearized equation of (18) as follows:

$$
\begin{aligned}
\dot{\mathbf{x}}^{c} & =\mathbf{A} \mathbf{x}^{c}(t)+\mathbf{B} \mathbf{u}^{c}(t)+\mathbf{A}_{l} \mathbf{x}^{c}(t)+\mathbf{B}_{l} \mathbf{u}^{c}(t) \\
& =\left(\mathbf{A}+\mathbf{A}_{l}\right) \mathbf{x}^{c}(t)+\left(\mathbf{B}+\mathbf{B}_{l}\right) \mathbf{u}^{c}(t)
\end{aligned}
$$

Let $\mathbf{A}^{\prime}=\mathbf{A}+\mathbf{A}_{1}, \mathbf{B}^{\prime}=\mathbf{B}+\mathbf{B}^{\prime}$. Furthermore, one may obtain a linearized equation of (23) as follows:

$$
\dot{\mathbf{x}}^{c}=\mathbf{A}^{\prime} \mathbf{x}^{c}(t)+\mathbf{B}^{\prime} \mathbf{u}^{c}(t)
$$

Note that the feature matrix $\mathbf{C}_{p}$ is a fixed matrix corresponding to a unique location of hybrid systems. This means that solution of (18) will produce a unique continuous state $\mathbf{x}^{c}(t)$ for a given continuous state $\mathbf{x}^{c}(t)$ and control inputs $\mathbf{u}^{c}(t)$. Furthermore, linearized equation of (18) will produce the same conclusion. One may obtain a linearized model as follows:

$$
\begin{gathered}
\dot{\mathbf{x}}^{c}=\mathbf{A}^{\prime} \mathbf{x}^{c}(t)+\mathbf{B}^{\prime} \mathbf{u}^{c}(t) \\
\mathbf{x}^{d}(t)=\mathbf{b}_{d}\left(\mathbf{C}_{p}\right) \\
\mathbf{E}_{1} \mathbf{u}^{c}(t)+\mathbf{E}_{2} \mathbf{C}_{p}+\mathbf{E}_{3} \mathbf{x}(t) \leq \mathbf{E}_{4}
\end{gathered}
$$

The feature matrix $\mathbf{C}_{p}$ is a fixed matrix corresponding to a unique location. Here, the linearized model (25) - (27) just is the linear model of each location, not the linear model of the nonlinear hybrid system.

Let $l \in L$, where $L$ is the set of locations of the nonlinear hybrid system, (25) - (27) have got the linearized model for every location of nonlinear hybrid system. Finally, we get the linearized model of a nonlinear hybrid system by using the linearized model of every location. Summarize the process as the following theorem.

Theorem 5.1 A nonlinear hybrid system is linearized by its hybrid states' feature matrix as follows:

$$
\begin{aligned}
& \dot{\mathbf{x}}_{l}^{c}=\mathbf{A}_{l}^{\prime} \mathbf{x}_{l}^{c}(t)+\mathbf{B}_{l}^{\prime} \mathbf{u}_{l}^{c}(t) \\
& \mathbf{x}_{l}^{d}(t)=\mathbf{b}_{l d}\left(\mathbf{C}_{l p}\right)
\end{aligned}
$$

$$
\mathbf{E}_{l l} \mathbf{u}_{l}^{c}(t)+\mathbf{E}_{l 2} \mathbf{C}_{l p}+\mathbf{E}_{l 3} \mathbf{x}_{l l}(t) \leq \mathbf{E}_{l 4}
$$

Where $\mathbf{x}_{l}^{c}(t), \mathbf{u}_{l}^{c}(t)$ are the continuous state and discrete inputs of location $l$ respectively; $\mathbf{x}_{l}^{d}(t)$ is the discrete state of location $l$, the elements of $\mathbf{b}_{l d}$ depend on the status of the feature matrix $\mathbf{C}_{l p} ; \mathbf{E}_{l i}(i=1,2,3,4)$ are the corresponding coefficient matrices of appropriate size for location $l$.

\section{CONCLUSIONS}

A model framework of nonlinear hybrid systems is proposed which is based on the feature matrix of truth table, which simplifies the linearized computing. Furthermore, we consider a special nonlinear hybrid system and also develop the linearized model of it. Linearized a nonlinear hybrid system depends on its kinds, and our study is pure research. The model of nonlinear hybrid systems is a key performance consideration of systems. Our future work will focus on these two issues and proceed in three steps: firstly, developing methods to linearize more kinds of nonlinear hybrid systems; secondly, solving the computational problems of nonlinear hybrid systems; thirdly, putting this theory to some practical problems.

\section{ACKNOWLEDGMENT}

This work is supported by the Talent Introduction Project of Kunming University of Science and Technology, and the Natural Science Foundation of Guangxi under Grant No.2014GXNSFAA118359.

\section{REFERENCES}

[1] Branicky M S, Borkar V S, Mitter S K. A unified framework for hybrid control: model and optimal control theory[J]. Automatic Control IEEE Transactions on, 1998,43(1):31- 45.

[2] Bemporad A, Morari M. Control of systems integrating logic, dynamics, and constraints [J]. Automatica, 1999,35(3):407-427.

[3] Bemporad A, Morari M. Optimization-based hybrid control tools[C] American Control Conference, 2001. Proceedings of the 2001. IEEE, 2001:1689 - 1703.

[4] Alur R, Courcoubetis C, Henzinger T, et al. The algorithmic analysis of hybrid systems[J]. Theoretical Computer Science, 1995, 138(1):3-34.

[5] A. van der Schaft, H. Schumacher, An introduction to hybrid dynamical systems, Springer Verlag, London,2000.

[6] Sontag E D. Nonlinear regulation: The piecewise linear approach[J]. IEEE Transactions on Automatic Control, 1981, 26(2):346-358.

[7] W.P.M.H. Heemels, B. De Schutter, A. Bemporad. Equivalence of hybrid dynamical models [C]// Decision and Control, 2001. Proceedings of the, IEEE Conference on. IEEE, 2001:364-369 vol.1.

[8] Buss M, Glocker M, Hardt M, et al. Nonlinear Hybrid Dynamical Systems: Modeling, Optimal Control, and Applications[M]// Modelling, Analysis, and Design of Hybrid Systems. Springer Berlin Heidelberg, 2002:311-335.

[9] Nandola N N, Bhartiya S. A multiple model approach for predictive control of nonlinear hybrid systems[J]. Journal of Process Control, 2008, 18(2):131-148.

[10] Henzinger T A, Pei H H. Algorithmic analysis of non-linear hybrid systems[J]. IEEE Transactions on Automatic Control, 1998, 43(4):540554.

[11] Henzinger T A, Wong-Toi H. Linear phase-portrait approximations for nonlinear hybrid systems[C] Hybrid Systems Iii, Lncs. Springer Berlin Heidelberg, 1996:377-388.

[12] Branicky M S. Studies in hybrid systems : modeling, analysis, and control[J]. Massachusetts Institute of Technology, 1995. 
[13] Branicky M S. General Hybrid Dynamical Systems: Modeling, Analysis, and Control.[C] Hybrid Systems III: Veri cation and Control, Proceedings of the DIMACS/SYCON Workshop, October 22-25,1995, Ruttgers University, New Brunswick, NJ, USA.1995:186-200.

[14] Alamir M. Stabilization of Nonlinear System by Receding Horizon Control SCheme: A Parametrized Approach for Fast Systems[J]. 2006.

[15] Thomas W. Cusick, Pantelimon Stanica. Cryptographic Boolean function and applications[M]. Academic Press; 1st edition (March 27,2009).

[16] Glushkov V M. SYNTHESIS OF DIGITAL AUTOMATA[J]. Synthesis of Digital Automata, 1965.

[17] Glushkov V M, Kapitonova Yu V, Mishchenko A T, Discrete logic design [in Russian], Naukova Dumka,Kiev (1987).

[18] Zakrevskii A D, Cascade logic design [in Russian], Nauka, Moscow (1981).

[19] Pospelov D A, Logic methods of circuit analysis and design [in Russian], Energiya, Moscow (1974).

[20] Massey J L. A spectral characterization of correlation immune combining functions[J]. IEEE Transactions on Information Theory, 1988, 34(3):569-571.

[21] Qiaoyan Wen, xinyi Niu, yixian Yang, Modern cryptography Boolean functions [M]. Beijing: Science Press(2000).

[22] Branicky M S. Multiple Lyapunov functions and other analysis tools for switched and hybrid systems[J]. IEEE Transactions on Automatic Control, 1998, 43(4):475-482. 